\title{
Optical Absorption Enhancement in Thin Film Silicon Solar Sells: The Effect of Antireflection Coatings and Back-Reflectors
}

\author{
Sepideh Esmaeili Germezgholi ${ }^{a}$, Behrooz Rezaei ${ }^{a, *}$, and Sohrab Ahmadi-Kandjani ${ }^{a, b}$

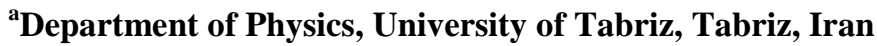 \\ ${ }^{b}$ Research Institute for Applied Physics and Astronomy, University of Tabriz, Tabriz, Iran \\ *Corresponding Author Email: rezaeibhr@yahoo.com
}

Regular paper: Received: Jun. 23, 2020, Revised: Oct. 22, 2020, Accepted: Jan. 3, 2021, Available Online: Jan. 7,2021, DOI: 10.29252/ijop.14.2.129

\begin{abstract}
In this paper, a thin film silicon solar cell with anti-reflection coatings on front surface and the combination of periodic grating and photonic crystal on its back surface has been considered. The thickness and number of anti-reflection coatings, as well as the geometric and physical parameters of photonic crystal and grating are optimized to increase the optical absorption of solar cell. The simulations have been performed using the finite difference time domain method with Lumercial software. The results show that the optical absorption of solar cell has been increased significantly by utilizing the anti-reflection coatings, photonic crystal and grating.
\end{abstract}

KEYWORDS: Photonic crystal, antireflection coating, silicon solar cell.

\section{I.INTRODUCTION}

Due to increase in air pollution and concentration caused by fossil fuels such as oil, gas, etc., and the gradual warming of the earth, tendency to use solar energy is increasing. Photovoltaic technology is one of the best ways to use solar energy and based on this, it is important to study solar cells as a clean source for producing energy [1]. Among all semiconductor solar cells, a large part of the photovoltaic market are devoted to silicon (Si) solar cells. To increase the absorption in thin $\mathrm{Si}$ layers, various techniques such as utilizing the anti-reflective coatings (ARC) on front surface of the solar cell and also utilizing metals, photonic crystals and grating as back reflectors, have been used [2]-[3]. Recently, some studies have been conducted on the simultaneous use of photonic crystals and grating as a back reflector in solar cells and it has been shown that this method has significant increase in absorption and thus efficiency of solar cells [4]-[5].The aim of this study is to use photonic crystal and grating as a back reflector of the thin film $\mathrm{Si}$ solar cell and optimize its geometrical and physical parameters in order to increase the absorption in the active layer of Si. Also, the type, thickness and number of ARC layers will be optimized to increase the absorption of the active Si layer.

\section{COMPUTATional METHOD}

The structure of the solar cell studied in this paper has been shown in Fig. 1. The active layer of the solar cell is $\mathrm{Si}$ with a thickness of $D$. The one-dimensional photonic crystal is composed of $\mathrm{SiO}_{2}$ and $\mathrm{InSb}$ dielectric layers with refractive index and thicknesses of $\left(n_{1}, d_{1}\right)$ and $\left(n_{2}, d_{2}\right)$, respectively. The grating is made of $\mathrm{SiO}_{2}$ and its diffraction characteristics are grating period, $\Lambda$, depth, $h$, and width, $b$, as shown in Fig. 1. The thickness of the ARC is optimized to get the lowest reflection from the solar cell structure. The thickness of ARC is equal to $t$, when it is a single layer, and for the case of two layers ARC, the thicknesses of layers are represented with $t_{1}$ and $t_{2}$, as obtained from the following relations [6]: 


$$
\begin{aligned}
& t=\frac{\lambda_{0}}{4 n} \\
& t_{1}=\frac{\lambda_{0}}{2 \pi n_{1}} \sqrt{\tan ^{-1}\left[\frac{\left(n_{s}-n_{0}\right)\left(n_{2}^{2}-n_{0} n_{s}\right) n_{1}^{2}}{\left(n_{1}^{2} n_{s}-n_{0} n_{2}^{2}\right)\left(n_{0} n_{s}-n_{1}^{2}\right)}\right]} \\
& t_{2}=\frac{\lambda_{0}}{2 \pi n_{2}} \sqrt{\tan ^{-1}\left[\frac{\left(n_{s}-n_{0}\right)\left(n_{0} n_{s}-n_{1}^{2}\right) n_{2}^{2}}{\left(n_{1}^{2} n_{s}-n_{0} n_{2}^{2}\right)\left(n_{2}^{2}-n_{0} n_{s}\right)}\right]}
\end{aligned}
$$

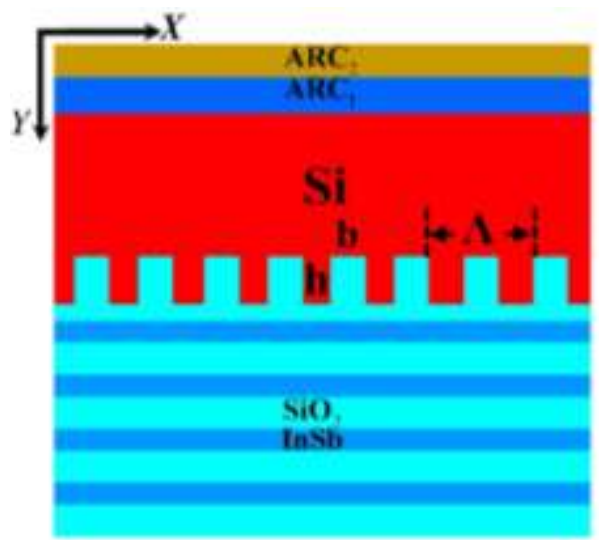

Fig. 1. Schematic representation of the studied solar cell structure.

In the above relations, $\lambda_{0}$ is the wavelength of the incident light in free space, $n_{0}, n_{1}, n_{2}$ and $n_{s}$ are the refractive index of the input medium, the first ARC layer, the second ARC layer and active $\mathrm{Si}$ layer at the this wavelength, respectively. In this paper, in order to calculate the absorption spectrum of the studied solar cell structure, the finitedifference time-domain method (FDTD) using the Lumerical software has been utilized [7][8]. The simulation area for the propagation of the TM-polarized light (electric field along the $\mathrm{Z}$ axis) has a periodic boundary condition in the lateral direction ( $\mathrm{X}$ direction) and the perfect matched layer (PML) absorbing boundary condition [9] is applied in the Y direction. The dimensions of the computing area are $0.86 \mu m \times 6 \mu m$ and the spatial steps are considered as $\Delta x=\Delta y=0.25 \mathrm{~nm}$.

\section{III.RESULTS AND DISCUSSION}

The structure of the solar cell includes a thin layer of active $\mathrm{Si}$ with thickness of $D=2 \mu \mathrm{m}$.
To begin, we obtain the absorption spectrum of the Si without coating and back-reflector structures, called reference solar cell. The corresponding absorbance spectrum is shown in Fig. 2 in black solid curve. It should be noted that the simulation was performed with Lumerical software and the obtained data for absorption spectra were plotted in Matlab software.

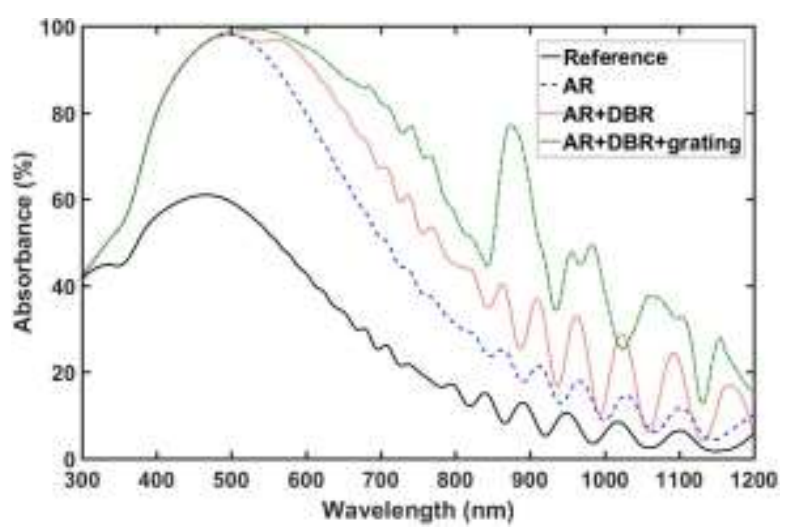

Fig. 2. The absorbance spectra of thin film Si solar cell with $\mathrm{SiO}$ single layer ARC at four cases: 1) Reference (without ARC, photonic crystal and grating), 2) with ARC, 3) with ARC and photonic crystal, and 4) with ARC, photonic crystal and grating, which are represented by solid, dashed, dotted and dashed-dotted lines, respectively.

Then, the effect of ARC is investigated on transmission spectrum of the reference solar cell. The transparent ARC layer is considered to reduce the reflection from the surface of the solar cell. Mostly, the materials $\mathrm{TiO}_{2}, \mathrm{SiO}_{2}$, $\mathrm{ZnO}, \mathrm{Al}_{2} \mathrm{O}_{3}$ and etc, are used as ARC in solar cells. In this paper, $\mathrm{SiO}$ is used as a single layer $\mathrm{ARC}$ and $\mathrm{ZnO} / \mathrm{TiO}_{2}, \mathrm{MgF}_{2} / \mathrm{TiO}_{2}$ and $\mathrm{Al}_{2} \mathrm{O}_{3} / \mathrm{TiO}_{2}$ are used as double layer ARC. First, we study the effect of single layer ARC on absorption spectrum of the solar cell. The $\mathrm{SiO}$ material with refractive index of $n_{S i O}=1.99$ at target wavelength $\lambda_{0}=525 \mathrm{~nm}$ [11] is considered as a single layer ARC, where its optimum thickness equals to $t=65.95 \mathrm{~nm}$, which is obtained from Eq. (1). The absorbance spectrum of thin film Si solar cell with ARC is shown in Fig. 2 in blue dashed curve. It is obvious that the absorbance of Si has been increased remarkably by adding the ARC on its front surface. 
In the next step, the photonic crystal as a backreflector structure is added to enhance the absorption of $\mathrm{Si}$ layer. The photonic crystal composed of 8-periods of $\mathrm{SiO}_{2}$ and $\mathrm{InSb}$ dielectric layers with refractive indices $n_{1}=1.46$ and $n_{2}=4.72$, respectively [10]. The thickness of the dielectric layers are set to be the quarter-wavelength of the materials, i.e., $d_{i}=\lambda_{c} / 4 n_{i}(i=1,2), \quad \lambda_{c} \quad$ is the central wavelength. In Fig. 3, the transmitance spectra of the photonic crystal at different central wavelengths are plotted. The numerical calculations show that the transmittance spectrum of the photonic crystal represents a very large photonic band gap at $\lambda_{c}=750 \mathrm{~nm}$ and varies in the wavelength range of 550$1200 \mathrm{~nm}$. Therefore, the more fraction of the AM $1.5 \mathrm{G}$ solar spectrum intensity distribution in wavelength range of $300-1200 \mathrm{~nm}$ can be reflected into the active $\mathrm{Si}$ layer due to the presence of this wider photonic band gap, which leads to increased absorption of $\mathrm{Si}$. As shown in Fig. 2, the absorbance of solar cell has been increased noticeably by adding the photonic crystal on its back surface, which is represented by red dotted curve.

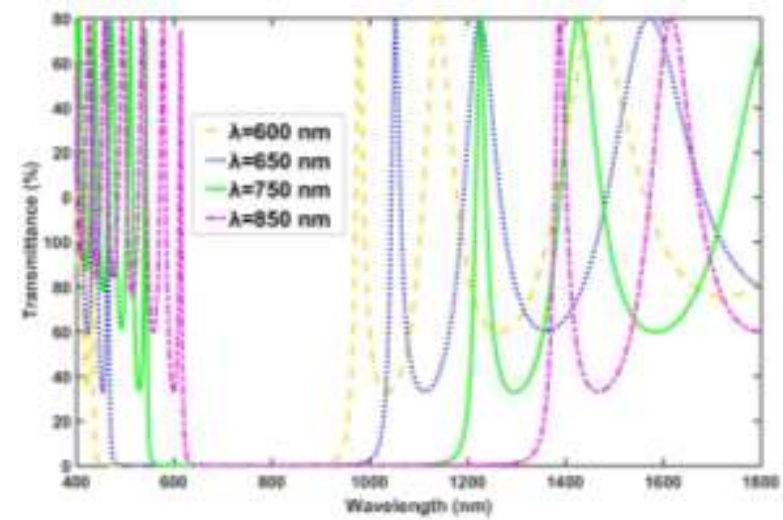

Fig. 3. The transmittance spectra of the photonic crystal at different central wavelengths.

Now, the parameters of the grating are optimized to improve the light trapping of $\mathrm{Si}$ solar cell. The diffraction grating is made of $\mathrm{SiO}_{2}$ and plays an important role in lighttrapping for our solar cells. To design the gratin structure, three parameters need to be considered: grating period, $\Lambda$, depth, $h$, and width, $b$. In Fig. 4 (a), we show the absorbance spectra of the solar cell at different values of $\Lambda$. In this simulation, we set the grating depth and width to be $h=0.31 \mu \mathrm{m}$ and $b=0.40 \mu \mathrm{m}$, respectively; both are optimal values, as we will show later. The optimal ARC and photonic crystal are used. It is obvious that the absorption of the solar cell is noticeable at $\Lambda=0.86 \mu \mathrm{m}$. Then, we investigated the effect of grating width on $\mathrm{Si}$ absorption at optimal values of $\Lambda=0.86 \mu \mathrm{m}$ and $h=0.31 \mu \mathrm{m}$. Figure 4(b) illustrates the absorbance spectra of Si solar cell for different values of grating width, $b$, at fixed value of above mentioned parameters. It can be seen that the absorbance is remarkable at $b=0.40 \mu \mathrm{m}$.
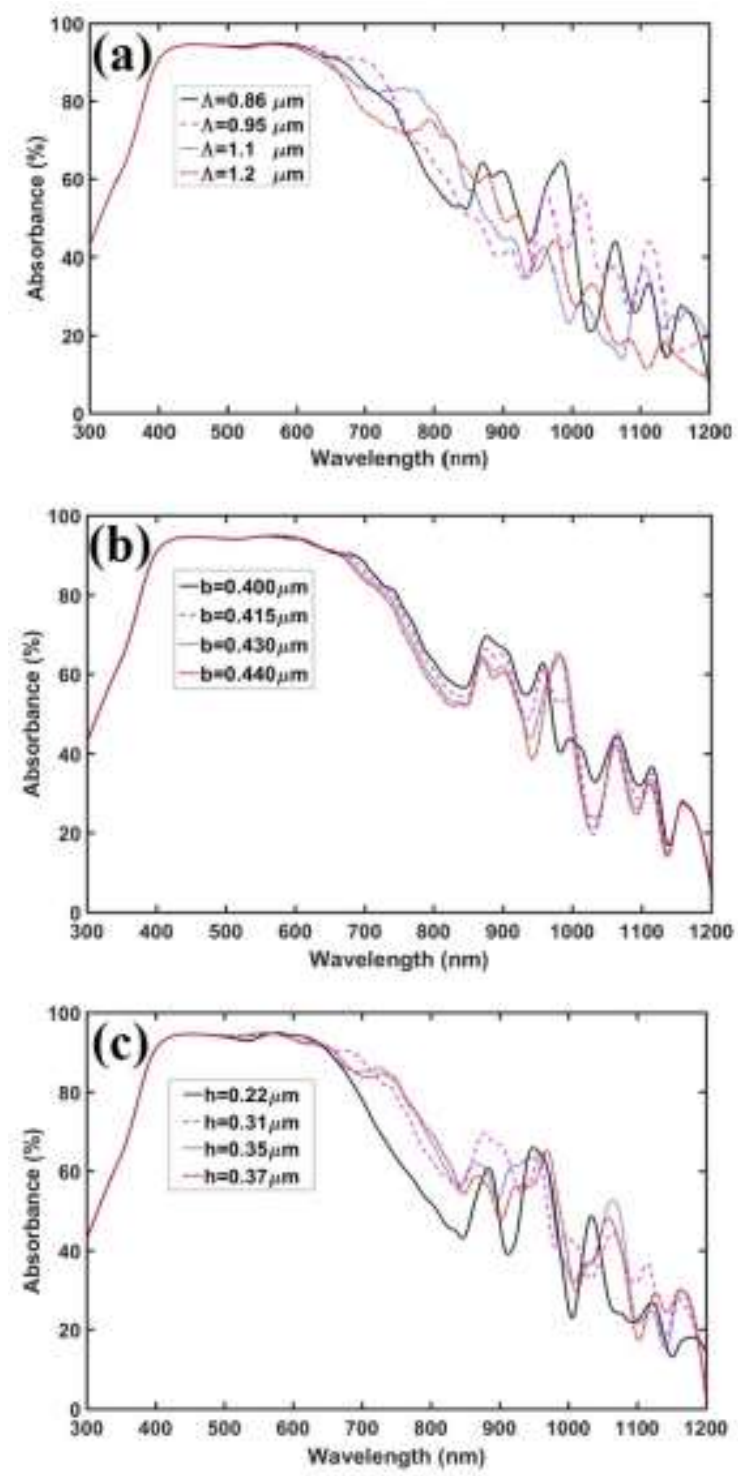

Fig. 4. The absorbance spectra of the Si solar cell for different values of (a) $\Lambda$ at fixed grating 
parameters $h=0.31 \mu \mathrm{m}$ and $b=0.40 \mu \mathrm{m}$, (b) $b$ at fixed grating parameters $\Lambda=0.86 \mu \mathrm{m}$ and $h=0.31 \mu m$, (c) $h$ at fixed grating parameters $\Lambda=0.86 \mu \mathrm{m}$ and $b=0.40 \mu \mathrm{m}$.

In addition, by selecting the optimal values for grating period, $\Lambda=0.86 \mu \mathrm{m}$, and width, $b=0.40 \mu \mathrm{m}$, the effect of grating depth, $h$, is investigated on optical absorption of Si solar cell. Figure 4(c) shows the absorbance spectra of the cell for different values of grating depth when the other optimal grating parameters are applied. The results indicate that the absorption of solar cell is remarkable at $h=0.31 \mu \mathrm{m}$. As Fig. 2 shows, the solar cell with optimal ARC, photonic crystal and grating structures represents very noticeable absorption, denoted by green dashed-dotted curve. It is clear that in all solar cell structures the trapping factors (ARC, photonic crystal and grating) lead to a significant increase in the optical absorption of the active Si layer and also the existence of ARC leads to noteworthy increase of absorption in lower frequency region.

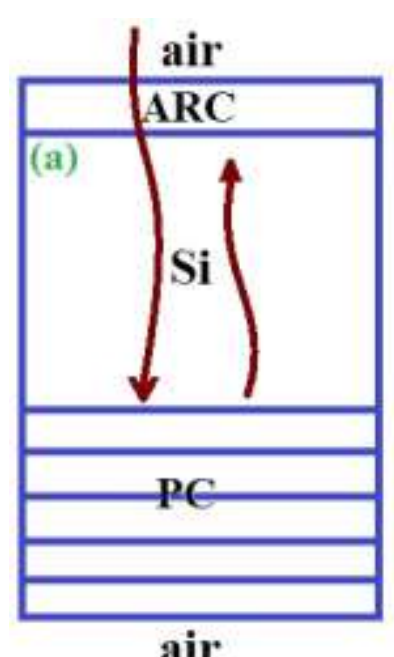

air

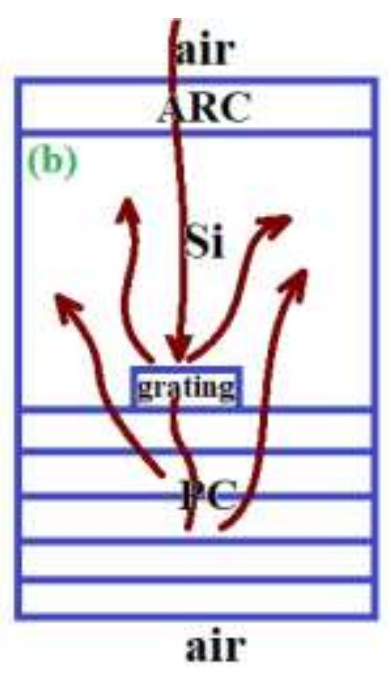

\section{air}

Fig. 5. Schematic representation of Si solar cell (a) without and (b) with diffractive grating on photonic crystal (PC).

To clarify the effect of grating on optical absorption of $\mathrm{Si}$, we have schematically compared the $\mathrm{Si}$ solar cell with and without grating on photonic crystal, as shown in Fig. 5. It is clear that the gratin diffracts light and therefore reflects back into the Si layer again, which consequently leads to the optical pathlength enhancement. Therefore, the diffractive grating plays an important role in optical absorption enhancement of Si layer.

In the next step, we consider two layers of ARC. In this case, the target wavelength is assumed to be $\lambda_{0}=750 \mathrm{~nm}$, because the wide range of wavelengths with least reflection can be achieved from the surface of the solar cell.

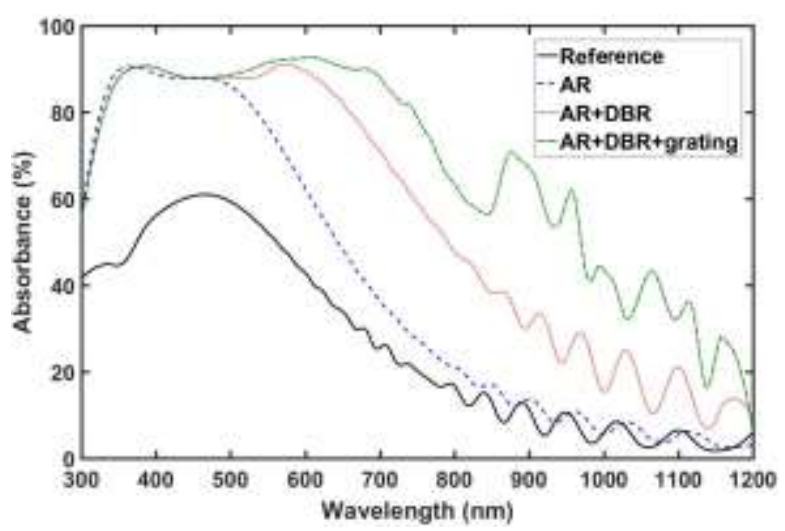

Fig. 6. The absorbance spectra of thin film Si solar cell with two coating layers of $\mathrm{ZnO} / \mathrm{TiO}_{2}$ at four cases: 1) Reference, 2) with ARC, 3) with ARC and photonic crystal, and 4) with ARC, photonic crystal and grating, which are represented by solid, dashed, dotted and dashed-dotted lines, respectively.

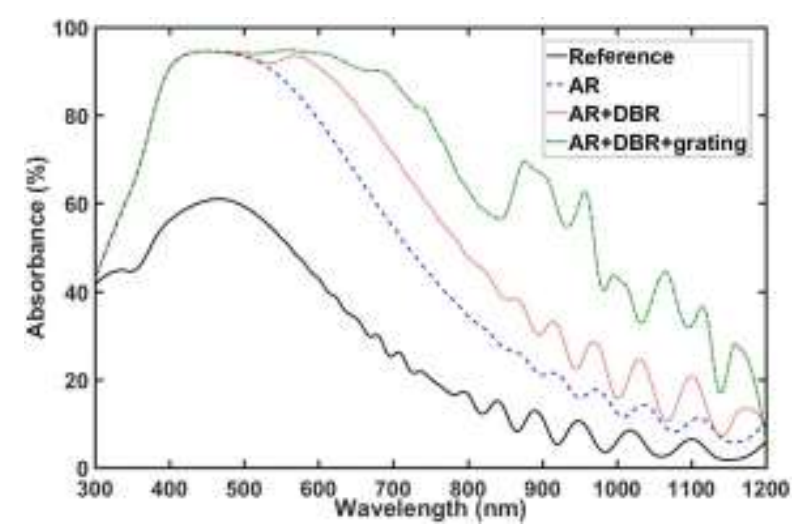

Fig. 7. The absorbance spectra of thin film Si solar cell with two coating layers of $\mathrm{MgF}_{2} / \mathrm{TiO}_{2}$ at four cases: 1) Reference, 2) with ARC, 3) with ARC and photonic crystal, and 4) with ARC, photonic crystal and grating, which are represented by solid, dashed, dotted and dashed-dotted lines, respectively.

For this target wavelength, the refractive index of ARC layers are $n_{\mathrm{TiO}_{2}}=2.35$, $n_{\mathrm{MgF}_{2}}=1.42, n_{\mathrm{ZnO}}=1.6$, and $n_{\mathrm{Al}_{2} \mathrm{O}_{3}}=1.76$ 
[11]. Therefore, according to Eq. (1), the thickness of two-layers anti-reflective coatings $\mathrm{ZnO} / \mathrm{TiO}_{2}, \quad \mathrm{MgF}_{2} / \mathrm{TiO}_{2}$ and $\mathrm{Al}_{2} \mathrm{O}_{3} / \mathrm{TiO}_{2}$ are $(76.15 \mathrm{~nm}, 50.88 \mathrm{~nm})$, $(87.30 \mathrm{~nm}, 61.68 \mathrm{~nm})$ and $(75 \mathrm{~nm}, 38.3$ $\mathrm{nm})$, respectively. The obtained results for absorbance spectra of the solar cell structures with two-layers ARC are shown in Figs. 6-8. Similar to previous structure, the absorbance spectra are calculated in four cases. Again, it can be observed that in all structures, the light trapping factors (ARC, photonic crystal and grating) have caused a significant increase in solar cell absorption, where the presence of double layer ARC leads to a significant increase in light absorption at low wavelength region. These figures show that the maximum absorption of solar cell structures with double coating layers of $\mathrm{ZnO} / \mathrm{TiO}_{2}$, $\mathrm{MgF}_{2} / \mathrm{TiO}_{2}$ and $\mathrm{Al}_{2} \mathrm{O}_{3} / \mathrm{TiO}_{2}$ are $92.73 \%$, $94.9 \%$, and $95.45 \%$, respectively.

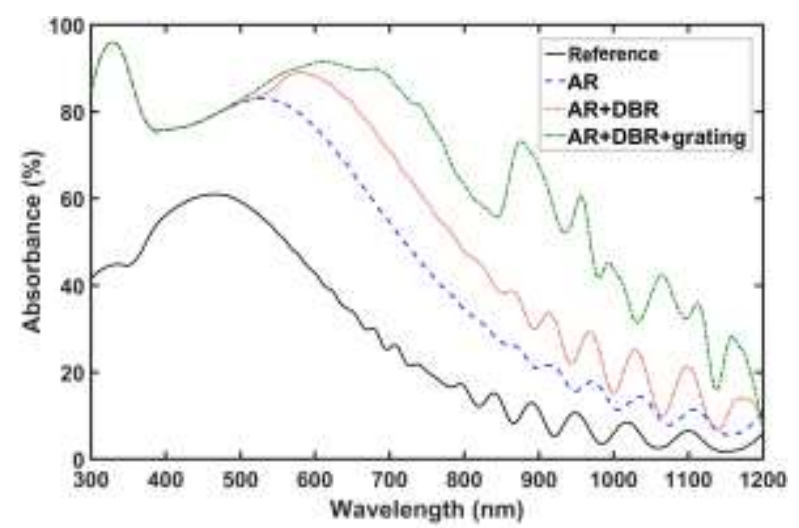

Fig. 8. The absorbance spectra of thin film Si solar cell with two coating layers of $\mathrm{Al}_{2} \mathrm{O}_{3} / \mathrm{TiO}_{2}$ at four cases: 1) Reference, 2) with ARC, 3) with ARC and photonic crystal, and 4) with ARC, photonic crystal and grating, which are represented by solid, dashed, dotted and dashed-dotted lines, respectively.

\section{IV.CONCLUSION}

In this paper, the thin film $\mathrm{Si}$ solar cell structures including anti-reflective coating layers on the front surface and onedimensional photonic crystal and grating, as back reflectors, were designed. The thickness and number of anti-reflective coating layers, also, the geometrical and physical parameters of back reflector materials were optimized to enhance the absorption of active Si layer. The obtained results show that the absorption of active $\mathrm{Si}$ layer increases significantly by utilizing photonic crystal, grating and antireflective coatings. Also, it is clear that the width of absorbance spectrum for solar cell with double layers ARC is larger than the case with single layer ARC.

\section{REFERENCES}

[1] A.R. Prasad and D. Jagadish, "Developments in Solar Pond Technology: A Literature Review," Proceedings of National Conference on Frontiers in Mechanical Engineering, Bhopal, Madhya Pradesh, Vol. 3, pp. 154-156, 2013.

[2] P. Bermel, C. Luo, L. Zeng, L.C. Kimerling, and J.D. Joannopoulos, "Improving thin-film crystalline silicon solar cell efficiencies with photonic crystals," Opt. Express, Vol. 15, pp.16986-17000, 2007.

[3] N.-N. Feng, J. Michel, L. Zeng, J. Liu, C.-Y. Hong, L. C. Kimerling, and X. Duan, "Design of highly efficient Light-Trapping Structures for Thin-Film Crystalline Silicon Solar Cells," IEEE Trans. Electron Devices, Vol. 54, pp. 1926-1932, 2007.

[4] J. G. Mutitu, S. Shi, C. Chen, T. Creazzo, A. Barnett, C. Honsberg, and D. W. Prather, "Thin film silicon solar cell design based on photonic crystal and diffractive grating structures," Opt. Express, Vol. 16, pp. 15238$15248,2008$.

[5] X. Sheng, L. Z. Broderick, and L. C. Kimerling, "Photonic crystal structures for light trapping in thin-film Si solar cells: Modeling, process and optimizations," Opt. Commu. Vol. 314, pp. 41-47, 2014.

[6] H. A. Macleod, Thin-Film Optical Filters, $3^{\text {rd }}$ Ed. Institute of Physics Publishing: Bristol, UK, 2001.

[7] F. Oskooi, D. Roundy, M. Ibanescu, P. Bermel, J. D. Joannopoulos, and S. G. Johnson, "Meep: A flexible free-software package for electromagnetic simulations by the FDTD method," Comput. Phys. Commun. Vol. 181, pp. 687-702, 2010. 
[8] Lumerical Inc. FDTD Solutions; http://www.lumerical.com/tcadproducts/fdtd/.

[9] J. Berenger, "A perfectly matched layer for the absorption of electromagnetic waves," J. of Computational Physics. Vol. 114, pp.185-200, 1994.

[10]S. K. Tripathy, "Refractive indices of semiconductors from energy gaps," Opt. Materials, Vol. 46, pp. 240-246, 2015.

[11]M. N. Polyanskiy, Refractive index database. https://refractiveindex.info.

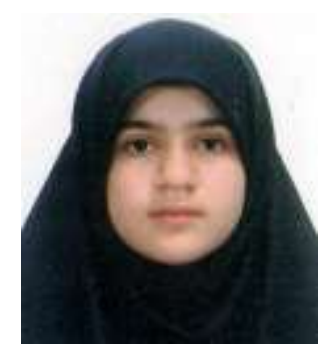

Sepideh Esmaeili Germezgholi received the B.Sc. degree in nuclear physics from Payame Noor University, Tabriz, Iran in 2016 and M.Sc. degree in Photonics-Electronics from University of Tabriz, Tabriz, Iran, in 2020. His current research interest includes the design of photonic crystals-based solar cells.

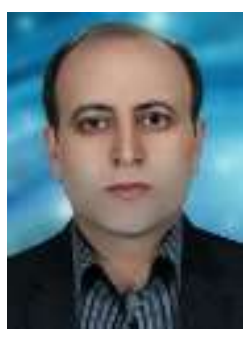

Behrooz Rezaei received the B.Sc. degree in 1998, the M.Sc. degree in 2001, and the Ph.D. degree in 2014, all from the University of Tabriz, Tabriz, Iran, in solid state physics. Since January 2001, he has been with the University of Tabriz, Tabriz, Iran, as an
Assistant Professor with the Research Institute for Applied Physics and Astronomy Department. In 2020, he joined the Department of Physics, University of Tabriz, as an Assistant Professor. His current research interests include the design and analysis of photonic crystals for the realization of cavities/waveguides, slow light structures, all optical switching devices, tunable photonic elements, graded index optics, and photonic crystals-based solar cells.

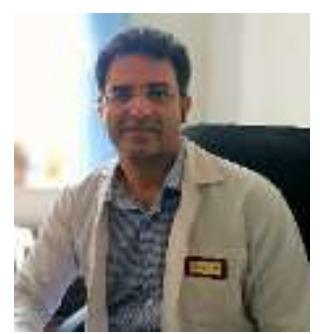

Sohrab Ahmadi-Kandjani was born in Tabriz, Iran, in 1972. He received the B.Sc. and M.Sc. degrees from the University of Tabriz, Iran, in 1996 and 1998, respectively, and the Ph.D. degree in physics from the University of Angres, France, in 2007. Since 1998, he has been a Faculty Member with the Faculty of Physics and Research Institute for Applied Physics and Astronomy (RIAPA), University of Tabriz, where he is currently the Head. He has authored or coauthored more than 100 scientific papers in peer-reviewed international journals. His experimental and theoretical research activities encircle the study of nonlinear optical effects in organic and organic-inorganic perovskite materials, surface relief gratings formation in azo-polymers, selforganized patterns in optics, organic and organic-inorganic perovskite optoelectronic devices, luminescent solar concentrators, computational ghost-imaging, optical sensors based on photonic crystals, SPRs, and LSPRs. 